\title{
Low-temperature solid-state reduction approach to highly reduced titanium oxide nanocrystals
}

\author{
Yoshihiro TSUJIMOTo ${ }^{1, \dagger}$ \\ ${ }^{1}$ Research Center for Functional Materials, National Institute for Materials Science, 1-1 Namiki, Tsukuba, Ibaraki 305-0044, Japan
}

Considerable attention has been paid to reduced transition metal nanomaterials because they exhibit attractive properties for practical applications in fuel cells, memory or thermoelectric devices, and photocatalysts. In particular, a series of oxygen-nonstoichiometric titanium oxides has advantages such as abundance of the elements, non-toxicity, corrosion resistance, and high electrical conductivity. However, the control of nanostructures is not straightforward because a high-temperature reductive condition is required for the synthesis of highly reduced metal oxides. Recently, it has been demonstrated that the novel low-temperature reduction technique can afford highly reduced titania nanoparticles with different particle sizes ranging from 20 to $300 \mathrm{~nm}$ diameter. This review article is focused on the synthesis of corundum-type $\mathrm{Ti}_{2} \mathrm{O}_{3}$ nanoparticles, the reaction mechanism, and the correlation between structure and physical properties.

(02018 The Ceramic Society of Japan. All rights reserved.

Key-words : Transition metal oxides, Nanocrystals, Low-temperature reduction, $\mathrm{Ti}_{2} \mathrm{O}_{3}$, Metal-insulator transition

\section{Scope and policy}

\section{Introduction}

Band gap insulators based on early transition metals, such as $\mathrm{TiO}_{2}$ and $\mathrm{WO}_{3}$, are of great interest because of their practical applications in photocatalysts, Li-ion battery anodes, pigments, and dielectric devices. ${ }^{1)-3)}$ Interestingly, oxygen removal from these insulators, which involves complex structure modification, drastically changes the physical properties. One of the most important examples is the family of $\mathrm{Ti}_{n} \mathrm{O}_{2 n-1}(2 \leq n)$, which has been extensively investigated over the last half century. ${ }^{4)-6)}$ The phase with $4 \leq n \leq 10$ is termed the Magnèli phase. $\mathrm{Ti}_{2} \mathrm{O}_{3}(n=2)$ is isostructural with corundum $\alpha-\mathrm{Al}_{2} \mathrm{O}_{3}$. As shown in Fig. 1, the rutile structure is built up with one-dimensional chains of edge-sharing $\mathrm{TiO}_{6}$ octahedra linked with another chains by sharing corners. In the corundum structure, each Ticentered octahedron shares one face along the $c$ axis and three edges in the $a b$ plane with each other. $\mathrm{Ti}_{n} \mathrm{O}_{2 n-1}$ ( $4 \leq n \leq 10)$ has rutile-like slabs with $n$ octahedral thickness, each of which is connected by corner- and facesharing. These oxygen-deficient phases exhibit high electrical conductivity at room temperature, which provides good opportunities for a new class of titania-based electronic devices, such as electrodes of fuel cells and resis-

$\dagger$ Corresponding author: Y. Tsujimoto; E-mail: TSUJIMOTO. Yoshihiro@nims.go.jp

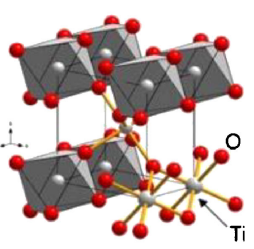

$\mathrm{TiO}_{2}$

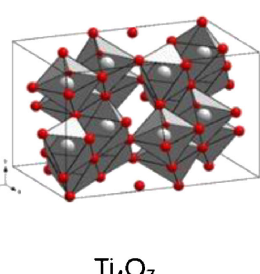

$\mathrm{Ti}_{4} \mathrm{O}_{7}$

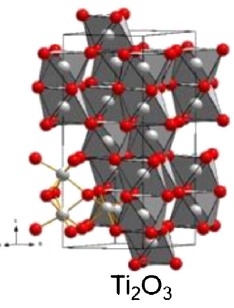

Fig. 1. Crystal structure of $\mathrm{TiO}_{2}, \mathrm{Ti}_{4} \mathrm{O}_{7}$, and $\mathrm{Ti}_{2} \mathrm{O}_{3}$.

tance random access memory. ${ }^{7-9)}$ For their practical applications, it is crucial to control the nanostructures of reduced titania. However, high-temperature reducing conditions are required to prepare highly reduced titania, which results in breaking of the nanostructures.

In this review paper, a novel approach to nanostructured reduced titania is presented: a low-temperature reduction method using binary metal hydride as a reducing agent. This reaction method was employed for the first time by Hayward and Rosseinsky in 1999 to synthesize $\mathrm{LaNiO}_{2}$ with a square planar $\mathrm{NiO}_{4}{ }^{10)}$ Metal hydrides such as $\mathrm{NaH}$ and $\mathrm{CaH}_{2}$ exhibit strong reducing power even at temperatures as low as $500^{\circ} \mathrm{C}$, which allows us to design in a topotactic manner complex metal oxides with unusual coordination environments. I successfully synthesized $\mathrm{Ti}_{2} \mathrm{O}_{3}$ of nanoparticles and nanorods by the reaction of $\mathrm{TiO}_{2}$ nanocrystals with $\mathrm{CaH}_{2}$ and systematically investigated the effects of size reduction on their structural and physical properties. ${ }^{11), 12)}$ 


\section{Low-temperature reduction for oxygen- deficient titanium oxide nanoparticles}

Commercially available $\mathrm{TiO}_{2}$ nanoparticles (average diameter: $20 \mathrm{~nm}$ ) with rutile structure (space group, $P 4_{2} /$ mnm) were reduced to $\mathrm{Ti}_{2} \mathrm{O}_{3}$ with corundum structure (R3-c) by reaction with $\mathrm{CaH}_{2}$ powder, maintaining the nanomorphology. ${ }^{11)} \mathrm{TiO}_{2}$, which was preheated at $200^{\circ} \mathrm{C}$ for several hours, was thoroughly mixed with $\mathrm{CaH}_{2}$ in a molar ratio of 1:4 in an Ar-filled glovebox. The mixture was pressed into a pellet, sealed in a Pyrex tube under vacuum without exposure to air, and then heated at $350^{\circ} \mathrm{C}$ in a tubular furnace for several days with intermediate grindings. Finally, the samples were washed with a $0.1 \mathrm{M}$ solution of $\mathrm{NH}_{4} \mathrm{Cl}$ in methanol to remove residual $\mathrm{CaH}_{2}$ and the byproduct $\mathrm{CaO}$. After filtration, bluish black powders were obtained (see Fig. 2). Note that 'bulk' $\mathrm{Ti}_{2} \mathrm{O}_{3}$ prepared at elevated temperatures is dark brown. Complete removal of calcium species was confirmed by energy dispersive X-ray analysis.

Figure 3 shows the room-temperature synchrotron X-ray diffraction (SXRD) patterns of the rutile $\mathrm{TiO}_{2}$ precursor and the product reduced for 4 or 10 days, measured using a Debye-Scherrer camera installed on BL15XU at SPring- 8 at a wavelength of $0.65298 \AA$. After reaction for 4 days, the SXRD patterns drastically changed: the rutile phase fully disappeared and new peaks appeared. The major peaks of the product can be readily indexed to a hexagonal cell $[a=5.0826(3) \AA, c=13.6864(14) \AA, V=$ $\left.306.19(4) \AA^{3}\right]$ with the space group $R 3-c$, which is similar to $\mathrm{Ti}_{2} \mathrm{O}_{3}$ structure. $\left.{ }^{6}\right)$ Compared with the lattice constants of bulk $\mathrm{Ti}_{2} \mathrm{O}_{3}[a=5.15443(1) \AA, c=13.60810(2) \AA, V=$ $\left.313.1051(7) \AA^{3}\right]$, the $a$ axis is contracted and the $c$ axis is

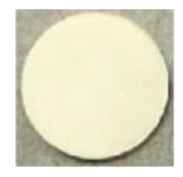

$\mathrm{TiO}_{2}$ nanoparticle

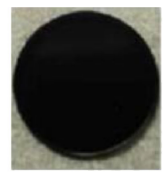

$\mathrm{Ti}_{2} \mathrm{O}_{3}$ nanoparticle

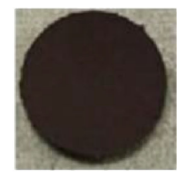

$\mathrm{Ti}_{2} \mathrm{O}_{3}$
Fig. 2. Photographs of the pellets of 20-nm-diameter $\mathrm{TiO}_{2}, 20$ nm-diameter $\mathrm{Ti}_{2} \mathrm{O}_{3}$, and sintered $\mathrm{Ti}_{2} \mathrm{O}_{3}$. ${ }^{12}$ )

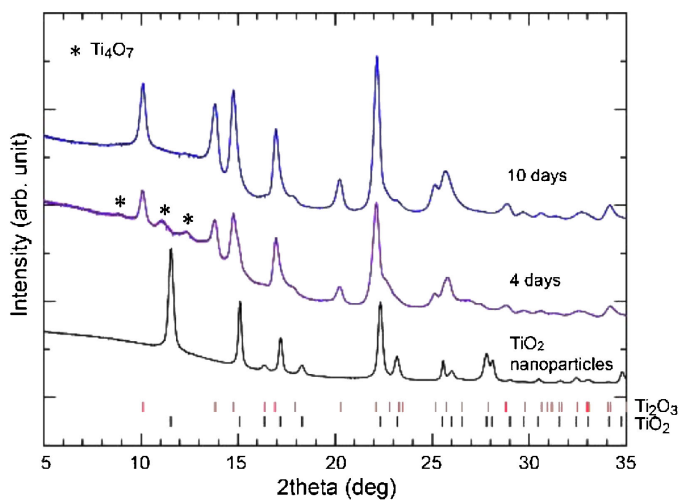

Fig. 3. Synchrotron X-ray powder diffraction patterns of rutile $\mathrm{TiO}_{2}$ nanoparticle and samples reduced for 4 or 10 days at $350^{\circ} \mathrm{C}^{11)}$ elongated. Several weak reflections could be assigned to $\mathrm{Ti}_{4} \mathrm{O}_{7}$ (space group $P-1$ ), a member of the Magnéli phase. The volume reduction involving the anisotropic volume change is attributed to the surface strain effects caused by particle-size reduction to a nanometric scale. In fact, transmission electron microscopy (TEM) observation revealed that no apparent particle growth occurred after the reduction [Figs. 4(a) and 4(c)]. In addition, the BrunauerEmmett-Teller (BET) measurements revealed that the product retained $80 \%$ of the specific surface area of the precursor $\left(80.72 \mathrm{~m}^{2} / \mathrm{g}\right)$. The high-resolution TEM (HRTEM) images of $\mathrm{TiO}_{2}$ and $\mathrm{Ti}_{2} \mathrm{O}_{3}$ nanoparticles are shown in Figs. 4(b) and 4(d), respectively, and the insets show selected area electron diffraction (SAED) patterns. The SAED patterns of the samples are consistent with the diffraction patterns expected from the rutile and corundum structures. The HRTEM images exhibit good crystallinity and uniformly spaced lattice fringes in each particle, indicating that each particle maintains the single-domain structure even after the reduction. These results are very surprising because the reductive reaction proceeds in a non-topotactic manner involving displacement of titanium atoms.

Next, the consequence of using anatase nanoparticles with $20 \mathrm{~nm}$ diameter and the surface area of $61.72 \mathrm{~m}^{2} / \mathrm{g}$ as a precursor was explored. Figure 5 shows the SXRD data of the products reduced at $350^{\circ} \mathrm{C}$ for 4,8 , or 12 days. As in the case of rutile, anatase reduced into $\mathrm{Ti}_{2} \mathrm{O}_{3}$ after 4 days, but the precursor remained in the products even after 8 days. No intermediate phases such as $\mathrm{Ti}_{4} \mathrm{O}_{7}$ are observed; however, the brookite phase formed together with $\mathrm{Ti}_{2} \mathrm{O}_{3}$, retaining the ratio of relative intensities. It is worth noting that the reduction of anatase with $\mathrm{CaH}_{2}$ involves particle growth. The specific surface areas reduced with increasing reaction time, for example, by $40 \%$ with respect to the original surface area after 12 days. This result suggests that not only the low-temperature treatment, but also the structural framework of the precursor are important factors for
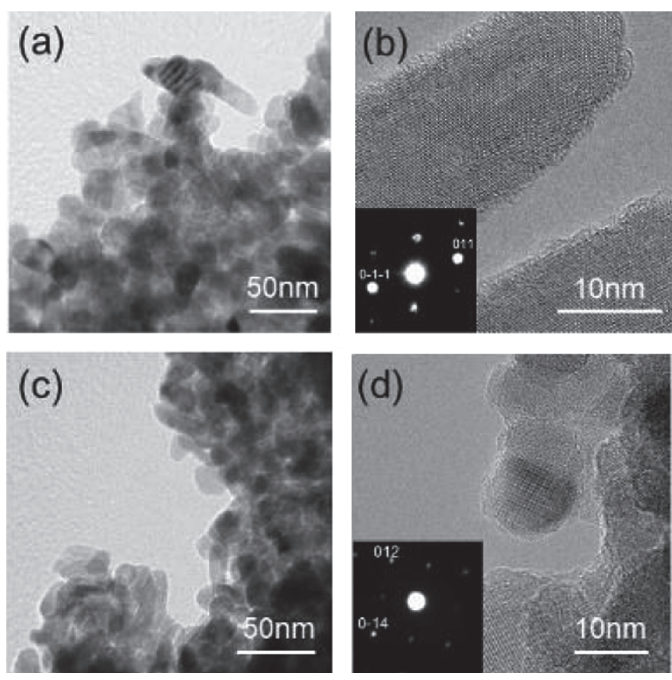

Fig. 4. TEM and HRTEM images of (a, b) $\mathrm{TiO}_{2}$ and (c, d) $\mathrm{Ti}_{2} \mathrm{O}_{3}$ nanoparticles. The insets of (b) and (d) are selected-area electron diffraction patterns. ${ }^{12}$ 


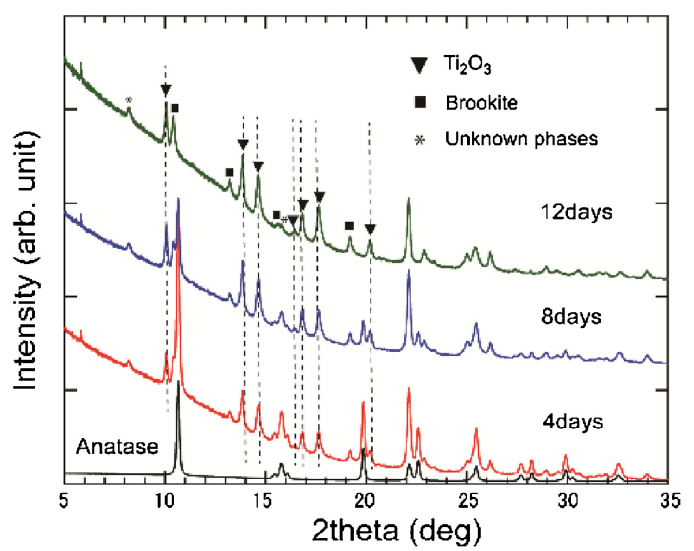

Fig. 5. Synchrotron X-ray powder diffraction patterns of anatase $\mathrm{TiO}_{2}$ nanoparticle and its reduced products.

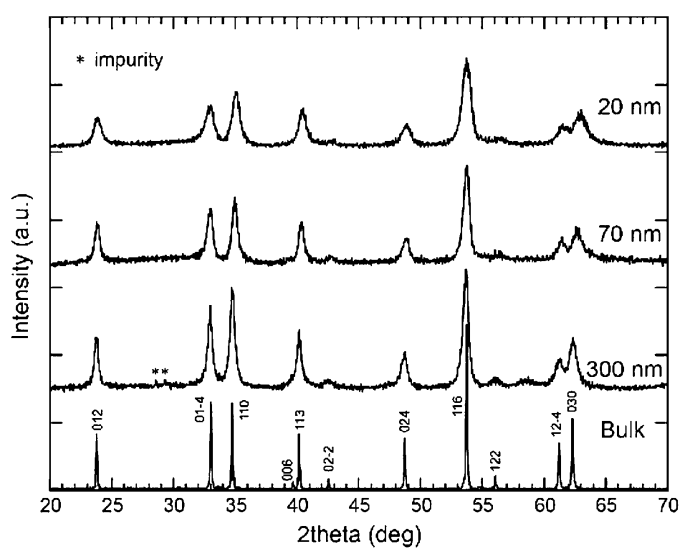

Fig. 6. X-ray powder diffraction patterns of $\mathrm{Ti}_{2} \mathrm{O}_{3}$ nanoparticles with different sizes. ${ }^{12)}$

maintaining the nanomorphology. As described above, $\mathrm{Ti}_{4} \mathrm{O}_{7}$, which is observed as an intermediate phase in the reaction of rutile with $\mathrm{CaH}_{2}$, has structural features common to both rutile and corundum structures. Thus, the formation of this phase plays a crucial role in suppressing the displacement of $\mathrm{Ti}$ atoms during the reductive reaction. Attempts were made to obtain $\mathrm{Ti}_{4} \mathrm{O}_{7}$ as a single phase by controlling the reaction time and temperature; however, mixed phases were always obtained with the precursor or corundum phase.

\section{Nanoparticles and nanorods with different sizes}

The low-temperature reduction is applicable to the synthesis of $\mathrm{Ti}_{2} \mathrm{O}_{3}$ nanoparticles and nanorods with different sizes. ${ }^{12)}$ Figure 6 shows the XRD patterns $(\mathrm{Cu} K \alpha)$ of the reduced products, which exhibit a broadening of the diffraction peaks with decreasing particle size. For nanoparticles with 70 and $300 \mathrm{~nm}$ diameters, the corundum phase is also obtained as the main phase; however, some lowintensity uncharacterized peaks appeared as minor phases for nanoparticles with $300 \mathrm{~nm}$ diameter. It should be noted that the reaction time increases with increasing particle size or surface area. Reduction with $\mathrm{CaH}_{2}$ proceeds through a solid-solid reaction between the reductant and precursors,

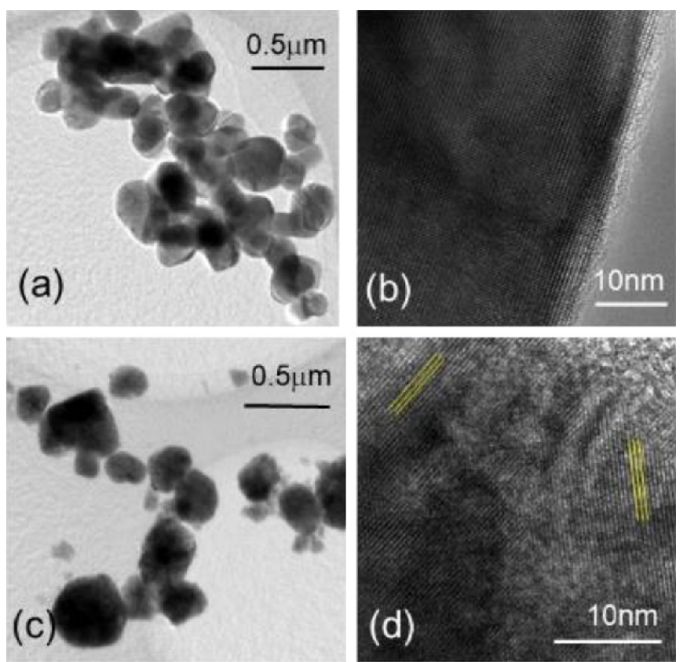

Fig. 7. TEM and HRTEM images of $(a, b)$ 300-nm-diameter $\mathrm{TiO}_{2}$ nanoparticles and (c, d) their reduced samples. ${ }^{12}$
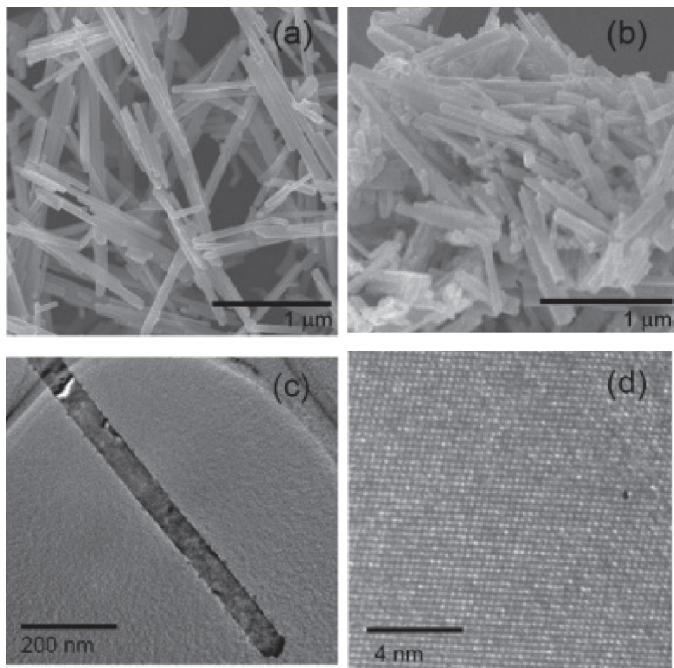

Fig. 8. SEM images of (a) $\mathrm{TiO}_{2}$ nanorods and (b) $\mathrm{Ti}_{2} \mathrm{O}_{3}$ nanorods. (c), (d) TEM and HRTEM images of the reduced nanorods.

and thus the physical contact between them is the key to promoting the reductive reaction. Indeed, $\mathrm{TiO}_{2}$ nanoparticles with 20 and $70 \mathrm{~nm}$ diameters and high surface areas were completely reduced to $\mathrm{Ti}_{2} \mathrm{O}_{3}$ within 8 days. However, the nanoparticles with $300 \mathrm{~nm}$ diameter and a much lower surface area $\left(6.36 \mathrm{~m}^{2} / \mathrm{g}\right)$ required 20 days.

Figure 7 shows the TEM and HRTEM images of $\mathrm{TiO}_{2}$ and $\mathrm{Ti}_{2} \mathrm{O}_{3}$ nanoparticles with $300 \mathrm{~nm}$ diameter, which reveals that the nanomorphology and particle size are no longer maintained. The $\mathrm{TiO}_{2}$ nanoparticles possess uniform size, spherical morphology, and a single-domain structure, while the nanoparticles after reduction broke into pieces, resulting in $21 \%$ increase in surface area. The HRTEM images of the reduced phase exhibit lattice fringes running in different directions between each other. These results are in marked contrast to those of 20-nm-diameter nanoparticles. The observed multi-domain formation can be accounted for by considering the drastic structural rearrangement. In relatively large particles, the stress from the 

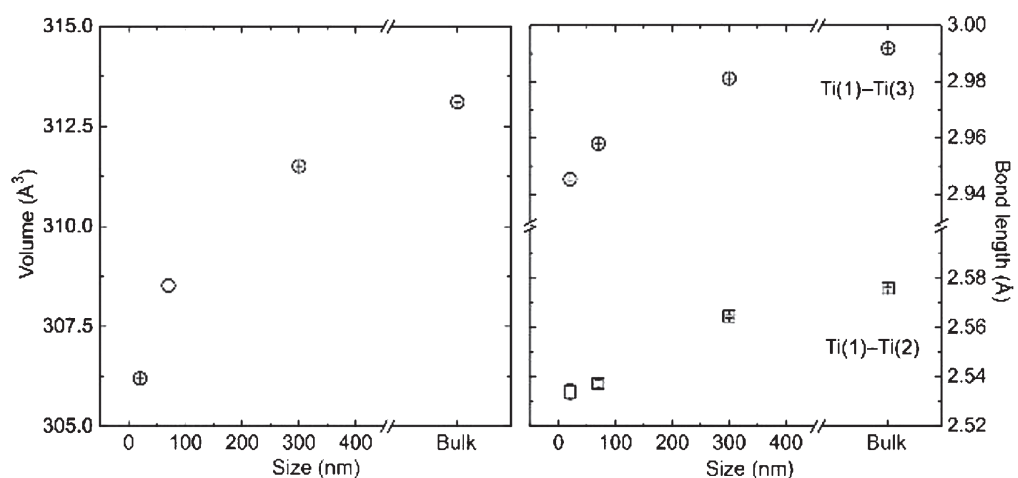

Fig. 9. Size dependence of volume and Ti-Ti bond lengths of $\mathrm{Ti}_{2} \mathrm{O}_{3}$ nanoparticles and bulk $\mathrm{Ti}_{2} \mathrm{O}_{3}$. ${ }^{12}$ )

displacement of $\mathrm{Ti}$ atoms and shearing motion is too great to maintain the single-domain structure of the precursor. The formation of multi-domain structures and broken particles results from the release of this stress energy.

$\mathrm{Ti}_{2} \mathrm{O}_{3}$ nanorods with $100 \mathrm{~nm}$ diameter and $\sim 1 \mu \mathrm{m}$ length can also be obtained as a single phase by the lowtemperature reduction. Figures 8(a) and 8(b) show the scanning electron microscopy (SEM) images of $\mathrm{TiO}_{2}$ and $\mathrm{Ti}_{2} \mathrm{O}_{3}$ nanorods, and Figs. 8(c) and 8(d) show the TEM and HRTEM images, respectively. These TEM images reveal that the single domain structure is maintained; however, the size and morphology are not maintained. As seen in the SEM images, the precursor is greater than $1 \mu \mathrm{m}$ in length, but the reductive reaction results in the breaking of the rods and roughing of the surfaces. The BET measurements reveal an increase in specific surface area from 10.6 to $13.2 \mathrm{~m}^{2} / \mathrm{g}$. These results are very similar to those of 300-nm-diameter nanoparticles. It should be noted that the morphological degradation observed in nanorods is caused by non-topotactic structural transformation, not by the grinding of $\mathrm{TiO}_{2}$ nanorods with $\mathrm{CaH}_{2}$ powders.

\section{Size dependence of structural and physical properties}

The successful synthesis of $\mathrm{Ti}_{2} \mathrm{O}_{3}$ nanoparticles with different sizes enables us to systematically investigate the effects of particle size on structural and physical properties. ${ }^{12)}$ Bulk $\mathrm{Ti}_{2} \mathrm{O}_{3}$ exhibits an unusual metal-to-insulator transition (MIT) in a wide temperature range from 420 (= $\left.T_{\text {MIT }}\right)$ to $550 \mathrm{~K}^{6}{ }^{6), 13), 14)}$ The sluggish transition of $\mathrm{Ti}_{2} \mathrm{O}_{3}$ is in sharp contrast to an abrupt MIT observed in $\mathrm{Ti}_{4} \mathrm{O}_{7}$ involving the charge ordering of $\left.\mathrm{Ti}^{3+} / \mathrm{Ti}^{4+} .{ }^{15}\right)$ Each $\mathrm{TiO}_{6}$ octahedron in $\mathrm{Ti}_{2} \mathrm{O}_{3}$ is subjected to a slight trigonal distortion, which results in the splitting of the three-fold degenerate $t_{2 \mathrm{~g}}$ orbitals into a low-lying $a_{1 \mathrm{~g}}$ orbital directed along the $c$ axis and two $e_{\mathrm{g}}^{\pi}$ orbitals directed toward the next-nearest neighbor Ti ions in the basal plane. ${ }^{16), 17)}$ The MIT involves a gradual elongation of the Ti-Ti dimer along the $c$ axis upon heating, which results in the narrowing of the energy gap between the bonding $a_{1 \mathrm{~g}}$ and anti-bonding $a^{*}{ }_{1 \mathrm{~g}}$ bands. Because the $e_{\mathrm{g}}{ }^{\pi}$ and $e_{\mathrm{g}}{ }^{* \pi}$ bands are located between the $a_{1 \mathrm{~g}}$ and $a^{*}{ }_{1 \mathrm{~g}}$ bands, the semiconducting gap between the $a_{1 \mathrm{~g}}$ and $e_{\mathrm{g}}{ }^{\pi}$ bands is closed across the MIT. ${ }^{16)}$

Given the strong correlation between the structure and

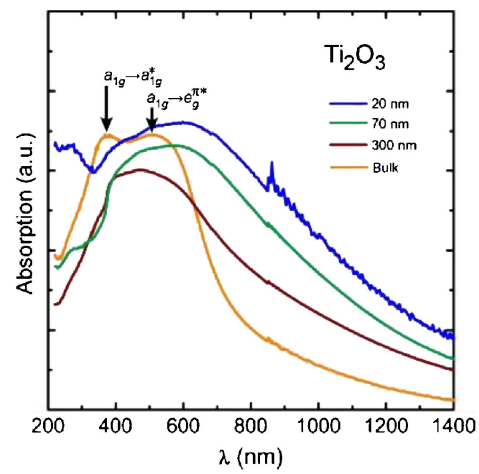

Fig. 10. UV-vis-NIR spectra of $\mathrm{Ti}_{2} \mathrm{O}_{3}$ nanoparticles and the bulk $\mathrm{Ti}_{2} \mathrm{O}_{3} .{ }^{12)}$

the electronic properties of $\mathrm{Ti}_{2} \mathrm{O}_{3}$, the particle-size reduction can be expected to affect the MIT. Figure 9 shows the particle-size dependence of the volume, and the bond lengths of the $\operatorname{Ti}(1)-\operatorname{Ti}(3)$ dimers in the basal plane and the Ti(1)-Ti(2) dimer along the $c$ axis. The volume and the intradimer distances monotonically shrunk with decreasing particle size. It is worth noting that the shrinkage of the $\mathrm{Ti}(1)-\mathrm{Ti}(2)$ bond increases the bandwidth of the $\mathrm{Ti} 3 \mathrm{~d}$ bands. This is confirmed from the ultraviolet visible near infrared (UV-vis-NIR) spectra shown in Fig. 10, where two peaks centered at 378 and $514 \mathrm{~nm}$ corresponding to the $a_{1 \mathrm{~g}}-a^{*}{ }_{1 \mathrm{~g}}$ and $a_{1 \mathrm{~g}}-e_{\mathrm{g}}{ }^{* \pi}$ interband transitions, ${ }^{18)}$ respectively, broadened with decreasing particle size. The increasing bandwidth leads to narrowing of the semiconducting gap. Figure 11 shows the temperature dependence of the electrical resistivity $(\rho)$ of 20 -nm-diameter sample and bulk $\mathrm{Ti}_{2} \mathrm{O}_{3}$. Although the $\rho$ of bulk $\mathrm{Ti}_{2} \mathrm{O}_{3}$ does not show any anomaly up to $400 \mathrm{~K},{ }^{19)}$ the $\rho$ vs $T$ curve for the nanoparticles exhibits a steep drop at $350 \mathrm{~K}$, which results from the onset of MIT. The reduction in $T_{\mathrm{MI}}$ by particle-size reduction is the first observation for $\mathrm{Ti}_{2} \mathrm{O}_{3}$.

\section{Summary}

Corundum $\mathrm{Ti}_{2} \mathrm{O}_{3}$ nanoparticles and nanorods with different sizes have been successfully synthesized by lowtemperature reduction using $\mathrm{CaH}_{2}$. The crystal structure, particle type and size of the parent materials play important roles in yielding homogeneous, high-quality $\mathrm{Ti}_{2} \mathrm{O}_{3}$ nanocrystals because of kinetics. Recently, similar tech- 


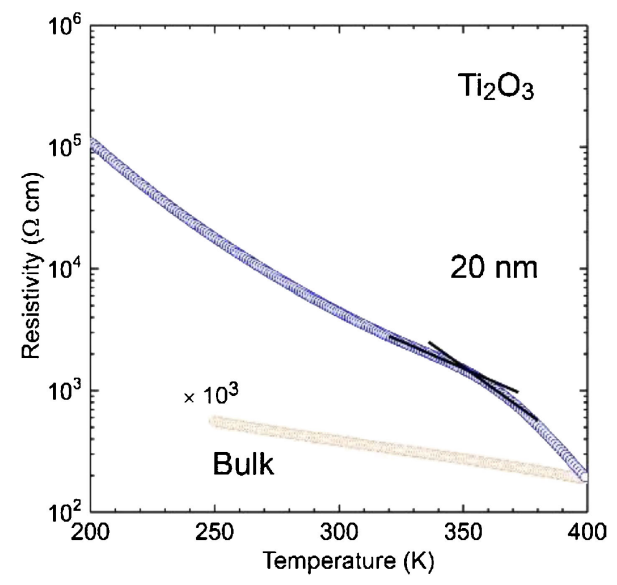

Fig. 11. Temperature dependence of the resistivity of $\mathrm{Ti}_{2} \mathrm{O}_{3}$ nanoparticles and bulk $\mathrm{Ti}_{2} \mathrm{O}_{3} .{ }^{12)}$

niques have been applied not only to nanoparticles, but also to thin films and core-shell structures. ${ }^{20)-22)} \mathrm{We}$ expect that the low-temperature reduction will open up the possibility of designing nanostructures of highly reduced phases, leading to new functional properties that cannot emerge in the bulk form.

Acknowledgement The author thanks all the collaborators for contributing to his studies. A part of our work was supported by JSPS KAKENHI (15K17837, 25289233, 50584075, 16H0648, 17K14466, 17H05493, 16H04501)

\section{References}

1) J. Pan, G. Liu, G. Q. Lu and H.-M. Cheng, Angew. Chem. Int. Edit., 50, 2133-2137 (2011).

2) D.-D. Qin, C.-L. Tao, S. A. Friesen, T.-H. Wang, O. K. Varghese, N.-Z. Bao, Z.-Y. Yang, T. E. Mallouk and C. A. Grimes, Chem. Commun., 48, 729-731 (2012).

3) K. J. Griffith, A. C. Forse, J. M. Griffin and C. P. Grey, J. Am. Chem. Soc., 138, 8888-8899 (2016).

4) L. A. Bursill and B. G. Hyde, Prog. Solid State Ch., 7, 177-253 (1972).

5) S. Andersson, A. Sundholm and Magnéli, Acta Chem. Scand., 13, 989-997 (1959).
6) C. E. Rice and W. R. Robinson, Acta Crystallogr. B, 33, 1342-1348 (1977).

7) S. Ohkoshi, Y. Tsunobuchi, T. Matsuda, K. Hashimoto, A. Namai, F. Hakoe and H. Tokoro, Nat. Chem., 2, 539545 (2010).

8) C. Yao, F. Li, X. Li and D. Xia, J. Mater. Chem., 22, 16560-16565 (2012).

9) D.-H. Kwon, K. M. Kim, J. H. Jang, J. M. Jeon, M. H. Lee, G. H. Kim, X.-S. Li, G.-S. Park, B. Lee, S. Han, M. Kim and C. S. Hwang, Nat. Nanotechnol., 5, 148-153 (2010).

10) M. A. Hayward, M. A. Green, M. J. Rosseinsky and J. Sloan, J. Am. Chem. Soc., 121, 8843-8854 (1999).

11) S. Tominaka, Y. Tsujimoto, Y. Matsushita and K. Yamaura, Angew. Chem. Int. Edit., 50, 7418-7421 (2011).

12) Y. Tsujimoto, Y. Matsushita, S. Yu, K. Yamaura and T. Uchikoshi, J. Asian Ceram. Soc., 3, 325-333 (2015).

13) C. E. Rice and R. Robinson, Mater. Res. Bull., 11, 1355-1360 (1976).

14) J. M. Honig and T. B. Reed, Phys. Rev., 174, 10201026 (1968).

15) M. Taguchi, A. Chainani, M. Matsunami, R. Eguchi, Y. Tanaka, M. Yabashi, K. Tamasaku, Y. Nishino, T. Ishikawa, S. Tsuda, S. Watanabe, C.-T. Chen, Y. Senba, H. Ohashi, K. Fujiwara, Y. Nakaura, H. Takagi and S. Shin, Phys. Rev. Lett., 104, 106401 (2010).

16) L. L. Van Zandt, J. M. Honig and J. B. Goodenough, J. Appl. Phys., 39, 594-595 (1968).

17) A. Mooradian and P. M. Raccah, Phys. Rev. B, 3, 42534256 (1971).

18) M. Uchida, J. Fujioka, Y. Onose and Y. Tokura, Phys. Rev. Lett., 101, 066406 (2008).

19) C. N. R. Rao, R. E. Loehman and J. M. Honig, Phys. Lett., 27A, 271-272 (1968).

20) A. Kitada, S. Kasahara, T. Terashima, K. Yoshimura, Y. Kobayashi and H. Kageyama, APEX, 4, 035801 (2011).

21) L. Seinberg, S. Yamamoto, R. Gallage, M. Tsujimoto, Y. Kobayashi, S. Isoda, M. Takano and H. Kageyama, Chem. Commun., 48, 8237-8239 (2012).

22) S. Yamamoto, G. Ruwan, Y. Tamada, K. Kohara, Y. Kusano, T. Sasano, K. Ohno, Y. Tsujii, H. Kageyama, T. Ono and M. Takano, Chem. Mater., 23, 1564-1569 (2011).

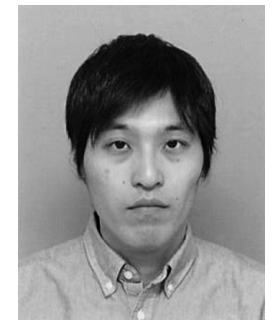

Yoshihiro Tsujimoto is a senior researcher of Research Center for Functional Materials, National Institute for Materials Science (NIMS), Tsukuba, Japan. He received his $\mathrm{PhD}$ in 2009 from Kyoto University. He was a JSPS fellow at Kyoto University from 2008 to 2010. He joined NIMS as a postdoctoral fellow from 2010 to 2012 and became a researcher in 2012. He was appointed as a senior researcher of NIMS in 2015. His research interests are in the anion-directed chemistry which yields unprecedented coordination environments around metal cations. 